\title{
Wann liegt nach neuester Rechtsprechung des EVG eine unfallähnliche Körperschädigung (UKS) vor - auch an der Rotatorenmanschette?
}

\author{
O. Niederberger ${ }^{a}$, K. Stutz ${ }^{b}$
}

\footnotetext{
a Rechtsabteilung, Suva, Luzern

b Ärzteteam Unfallmedizin, Suva, Luzern
}

* Urteil des EVG vom 5. Juni 2001 U 398/00.

Korrespondenz:

Dr. med. Klaus Stutz

SUVA

Fluhmattstrasse 1

Postfach 4358

CH-6002 Luzern
Im Jahr 2000 wurde an dieser Stelle der Artikel «Defekte der Rotatorenmanschette und unfallähnliche Körperschädigung» [1] publiziert. Ziel der Autoren war es, mit klaren, medizinisch breit abgestützten Kriterien zur Frage, wann eine Schädigung der Rotatorenmanschette eine unfallähnliche Körperschädigung sei, künftig Rechtsstreitereien zwischen Patienten, Versicherten und Versicherungen möglichst zu vermeiden helfen. Diese Kriterien wurden von der Arbeitsgruppe Schulter und der Arbeitsgruppe für Standesfragen der Schweizerischen Gesellschaft für Orthopädie (SGO) kritisch gewürdigt. Auf ihre Empfehlung hin unterstützte der Vorstand der SGO deren Verbreitung.

In Nr. 44 dieser Zeitschrift wurde die Diskussion zum Thema der unfallähnlichen Körperschädigung durch den Abdruck des Vortrags «Meniskusläsionen und soziale Unfallversicherung», den A. Bühler am SGO-Kongress vom 23. August 2001 gehalten hatte, weitergeführt [2].

Den Autoren dieser Mitteilung liegt daran, die Auswirkungen eines Urteils*, das Herr Bühler unter den Beispielen der vom EVG als unfallähnlich beurteilten Ereignisse zitierte, auch bezüglich der Rotatorenmanschettendefekte aufzuzeigen und so zur rechtsgleichen Beurteilung von allfälligen unfallähnlichen Körperschädigungen auch an der Schulter beizutragen.

Das EVG hat mit diesem Urteil entschieden, dass im Gegensatz zur Meinung der Unfallversicherer nicht die Schädigung unfallähnlich sein muss, sondern das Ereignis, und dass letzteres entscheidend dafür ist, ob eine Körperschädigung eindeutig auf eine Erkrankung oder Degeneration zurückzuführen ist oder nicht.

Am 5. Juni 2001 hatte das Eidgenössische Versicherungsgericht (EVG) zu beurteilen, ob bei einem 49jährigen Arbeiter eine unfallähnliche Körperschädigung vorliege. Dieser hatte im Anschluss an einen koordinierten Sprung von einer Kiste über einseitige Knieschmerzen geklagt, weswegen er einige Wochen später zum Arzt ging. Die Kniearthroskopie vier Monate nach dem Ereignis ergab eine Chondropathie Grad II mit herunterhängenden Knorpelfetz-

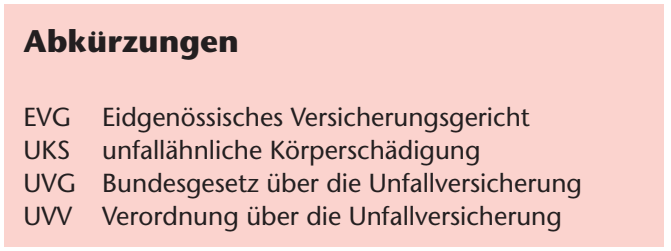

chen, korrespondierend mit einem Meniskusschaden, der im Hinterhorn durch einen degenerativen Längsriss entstanden war. Das Gericht urteilte (U 398/00) wie folgt:

Das Unfallversicherungsgesetz (UVG) führt im Bereich der unfallähnlichen Körperschädigungen notwendigerweise zu einer Verlagerung der Leistungspflicht von der Kranken- in die Unfallversicherung. Diese Folge wird bewusst in Kauf genommen, um die Problematik der Ausscheidung von Unfallund Krankheitsfolgen bei Gesundheitsschäden, in denen praktisch immer eine Vermischung unfall- und krankhafter bzw. degenerativer Einwirkungen vorliegt, zu vermeiden. Entscheidend ist bloss, dass ein äusseres, objektiv feststellbares, sinnfälliges Ereignis die Beschwerden verursacht oder zumindest ausgelöst hat. Da im konkreten Fall der als eindeutig degenerativ erkannte Meniskusriss nach einem Sprung von einer Kiste schmerzhaft wurde, liegt eine unfallähnliche Körperschädigung vor, wofür der Unfall- und nicht der Krankenversicherer haftet.

Das EVG definiert damit die unfallähnliche Körperschädigung («Körperschädigungen, die den Folgen eines Unfalles ähnlich sind» bzw. «Körperschädigungen, sofern sie nicht eindeutig auf eine Erkrankung oder eine Degeneration zurückzuführen sind») nicht über das pathoanatomische Substrat, sondern - zusammen mit der Diagnose allein über das unfallähnliche Ereignis. Nicht die Schädigung, sondern das Ereignis, bei dem sie sich manifestiert, muss unfallähnlich sein. Zitat: "Wo ein solches äusseres Ereignis mit Einwirkung auf den Körper nicht stattgefunden hat, und sei es auch nur als Auslöser eines in Art. 9 Abs. 
2 lit. a-h UVV aufgezählten Gesundheitsschadens, liegt eine eindeutig krankheits- oder degenerativ bedingte Gesundheitsschädigung vor».

Diese Rechtsprechung, die auf alle unfallähnlichen Körperschädigungen gemäss der Liste von Art. 9 Abs. 2 UVV übertragbar ist, hat zur Konsequenz, dass es belanglos ist, ob ein Meniskusschaden oder eine andere dort aufgeführte Schädigung morphologisch den typischen Folgen eines Unfalles entspricht. Der Arzt hat somit nur noch die Diagnose zu stellen. Sache der Versicherung bleibt es, das Vorliegen eines unfallähnlichen Ereignisses zu beurteilen.

In der im Dezember 2000 in dieser Zeitschrift erschienenen Arbeit «Defekte der Rotatorenmanschette und unfallähnliche Körperschädigung» [1] stellten die Autoren unter Bezugnahme auf eine seit Anfang 1998 in Kraft gesetzte Verordnungsbestimmung (Art. 9 Abs. 2 UVV) Kriterien zur Beurteilung der Frage auf, wann ein Rotatorenmanschettendefekt eindeutig krankhaften oder degenerativen Ursprungs ist. Die in dieser Arbeit gemachten Ausführungen haben aus medizinischer Sicht noch immer Gültigkeit. Sie sind aber mit dem EVG-Urteil vom 5. Juni 2001 (U 398/00) für den Entscheid, ob eine UKS vorliegt oder nicht, irrelevant geworden.

\section{Literatur}

1 Bär E, Stutz K, Gächter A, Gerber C, Zanetti M. Defekte der Rotatorenmanschette und unfallähnliche Körperschädigung. Schweiz Ärztezeitung 2000;81(49):2785-90.

2 Bühler A. Meniskusläsionen und soziale Unfallversicherung. Schweiz Ärztezeitung 2001;82(44): 2339-42.
Tabelle 1

Definition und Liste der UKS.

Art. 9 Abs. 2 UVV (gültig seit dem 01.01.98)

Folgende, abschliessend aufgeführte Körperschädigungen sind, sofern sie nicht eindeutig auf eine Erkrankung oder eine Degeneration zurückzuführen sind, auch ohne ungewöhnliche äussere Einwirkungen Unfällen gleichgestellt: ${ }^{17}$

a. ${ }^{18}$ Knochenbrüche;

b. Verrenkungen von Gelenken;

c. Meniskusrisse;

d. Muskelrisse;

e. Sehnenrisse;

g. Bandläsionen;

h Trommelfellverletzungen.

[17] Fassung gemäss Ziff. I der V vom 15. Dez. 1997 (AS 1998, 151).

[18] Fassung gemäss Ziff. I der V vom 15. Dez. 1997 (AS 1998, 151).

Tabelle 2

Merkmale des Unfalles und der UKS (Art. 9.1 und 9.2 UVV).

\begin{tabular}{lll} 
Merkmale & Unfall & UKS \\
1. schädigend & + & + \\
\hline 2. unbeabsichtigt & + & + \\
\hline 3. plötzlich & + & + \\
\hline 4. ungewöhnlich & + & 0 \\
\hline 5. äusserlich & + & +
\end{tabular}

